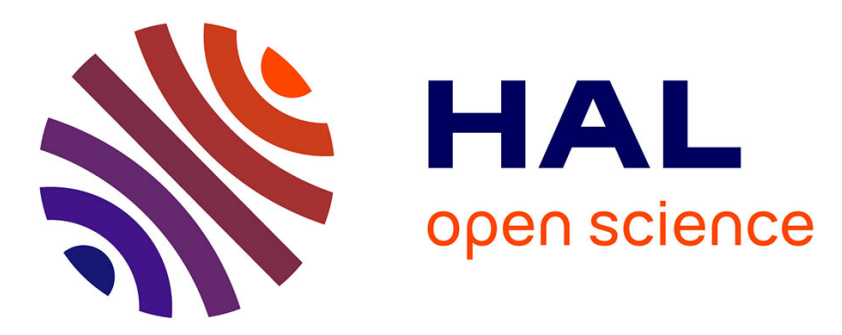

\title{
Validity of Laplace formula and dependence of surface tension on curvature in second gradient fluids
}

Francesco Dell'Isola, Giacomo Rotoli

\section{To cite this version:}

Francesco Dell'Isola, Giacomo Rotoli. Validity of Laplace formula and dependence of surface tension on curvature in second gradient fluids. Mechanics Research Communications, 1995, pp.6. hal-00498890

\section{HAL Id: hal-00498890 \\ https://hal.science/hal-00498890}

Submitted on 8 Jul 2010

HAL is a multi-disciplinary open access archive for the deposit and dissemination of scientific research documents, whether they are published or not. The documents may come from teaching and research institutions in France or abroad, or from public or private research centers.
L'archive ouverte pluridisciplinaire HAL, est destinée au dépôt et à la diffusion de documents scientifiques de niveau recherche, publiés ou non, émanant des établissements d'enseignement et de recherche français ou étrangers, des laboratoires publics ou privés. 


\section{VALIDITY OF LAPLACE FORMULA AND DEPENDENCE OF SURFACE TENSION ON CURVATURE IN SECOND GRADIENT FLUIDS.}

\section{F.dell'Isola and G.Rotoli ${ }^{\dagger}$.}

Dipartimento di Ingegneria Strutturale, Facoltà d'Ingegneria, Università di Roma "La Sapienza", Via Eudossiana 18, 00184 Roma ITALY.

(Received 7 June 1995; accepted for print 7 July 1995)

\section{Introduction}

Experiments on very small bubbles showed that classical Gibbs-Laplace theory on surface tension cannot be applied when the dimension of the interface becomes comparable with bubble radii [1]. On the other hand the pressure jump across the interface is from a experimental point of view the most suitable quantity to be measured in order to know surface tension and determine its dependence on curvature. Therefore Laplace formula needs to be generalized to the case of very small bubbles. The theory of second gradient fluids (Germain cf.[2]) accounts the effects of high density gradients (occurring in fluid interfaces) on fluid pressure. In [3] it is shown that second gradient theory allows the determination of the simplest consistent generalization of Laplace formula and an equivalent bubble theory in which surface tension and radius of a bubble are determined in terms of the density spatial field. This paper complements the quoted results analyzing them numerically. We find that our model: i) predicts the existence of a (minimal) nucleation radius, i.e. a radius which is a minimum possible for the equilibrium of a small bubble; ii) permits, through the new Laplace formula, to evaluate the dependence of surface tension on curvature with results very similar to actual experiments; iii) allows the determination of the range of validity of classical Laplace formula and a theoretical prediction on the departure of experimental data from it.

Equivalent bubbles in the theory of second gradient fluids.

In the theory of Laplace-Gibbs $[4,5]$ we have that the nucleation energy $w$ of bubbles of radius $R$ and surface tension $\sigma$ :

$$
w=4 \pi R^{2} \sigma+\frac{4}{3} \pi R^{3}\left[W\left(\rho_{v}\right)-W\left(\rho_{l}\right)-\mu\left(\rho_{l}\right)\left(\rho_{v}-\rho_{l}\right)\right]
$$


where $\rho_{l}$ and $\rho_{g}$ are respectively the densities of the liquid and of the vapor, $W$ is the free energy for unit volume and $\mu=\partial W / \partial \rho$ the chemical potential. The isothermal equilibrium conditions are:

$$
\mu\left(\rho_{l}\right)=\mu\left(\rho_{v}\right) \quad \mathcal{P}\left(\rho_{l}\right)-\mathcal{P}\left(\rho_{v}\right)=-\frac{2 \sigma}{R}
$$

where $\mathcal{P}=\rho \mu-W$ denote the thermodynamical pressure. As a consequence it is easy to prove that:

$$
w=\frac{4}{3} \pi R^{2} \sigma
$$

So that one can conclude that the nucleation energy of the bubble is one third of the creation energy of its interface. Second gradient theory, conceptually more straightforward than Gibbs-Laplace theory, can be used to construct a theory of capillarity. In such theory the internal mass energy $\epsilon$ is a function of the gradient of density $\nabla \rho$ as well as density $\rho$. The energy $\epsilon$ characterizes both the compressibility and capillarity properties of the fluid, independently of the bodies with which it is in contact. In the simplest models of second gradient fluids $[2,6,7,8]$ it is assumed that:

$$
\epsilon\left(\rho, \beta_{\rho}\right)=\epsilon_{0}(\rho)+\frac{\lambda}{2 \rho} \beta_{\rho}
$$

where $\lambda$ is the so-called capillary constant and $\beta_{\rho}=(\nabla \rho)^{2}$. In Eq. (4) the term $\frac{\lambda}{2 \rho} \beta_{\rho}$ is added to the energy of the classical compressible fluid (we note that the thermodynamical pressure is given by $\left.\mathcal{P}=\rho^{2} \epsilon_{0, \rho}\right)$. In absence of external forces the equilibrium equation is written: $\nabla \cdot \mathbf{S}=0$ where $\mathbf{S}$ is the general stress tensor:

$$
\mathbf{S}=-p \mathbf{I}-\lambda(\nabla \rho) \otimes(\nabla \rho)^{T}
$$

In (5) $\lambda$, accounts for the capillarity effects of $\nabla \rho$ in the equilibrium equation and $p=$ $\mathcal{P}-\lambda\left(\frac{1}{2} \beta_{\rho}+\rho \Delta \rho\right)$ is the mechanical pressure (which appears in boundary conditions [9]). Equilibrium equation in terms of $\rho$ becomes (cf. Rocard [9] and Blinowski [11]):

$$
\lambda \Delta \rho=\mu(\rho)-\mu\left(\rho_{l}\right)
$$

As the solution of Eq.(6) is the density profile $\rho(r)$ of the bubble, we call it the Density Profile Equation (DPE). In the theory of second gradient fluids [12], the nucleation energy $w$ takes into account also the capillary energy:

$$
w=\int_{D}\left[W(\rho)-W\left(\rho_{l}\right)-\mu\left(\rho_{l}\right)\left(\rho-\rho_{l}\right)+\frac{\lambda}{2}(\nabla \rho)^{2}\right] d v
$$

We deal here with bubbles which are small with respect to the size of the liquid-vapour interface phase. In this case the Gibbs results about the nucleation energy are found also using the theory of second gradient fluids [2]. Indeed it can be demonstrated using the DPE that:

$$
w=4 \pi \int_{0}^{\infty}\left[\psi(\rho)+\frac{\lambda}{2} \rho_{r}^{2}\right] r^{2} d r=\frac{4}{3} \pi \int_{0}^{\infty} \lambda \rho_{r}^{2} r^{2} d r=\frac{4}{3} \pi \bar{R}^{2} \int_{0}^{\infty} \lambda \rho_{r}^{2} d r
$$

where $\psi(\rho)=W(\rho)-W\left(\rho_{l}\right)-\mu\left(\rho_{l}\right)\left(\rho-\rho_{l}\right)$ and $\bar{R}^{2}$ is the mean value of $r^{2}$ with respect to the measure $\rho_{r}^{2} d r$. For a large bubble, i.e., when $\rho_{l}$ tends to plane interface value, $\bar{R}$ 
represents the radius. The surface tension for plane interface is $\int_{0}^{\infty} \lambda \rho_{\tau}^{2} d r$ (cf. [12]). Then Eq.(8) extends Gibbs relation to microscopic bubbles and reduces to it in the case of large bubbles. In second gradient theory stress tensor in the centre of a spherical bubble takes the value $\mathbf{S}=-p_{v} \mathbf{I}$ where $p_{v}=\mathcal{P}\left(\rho_{v}\right)-\lambda \rho_{v} \Delta \rho_{\rho=\rho_{v}}$. The value $\rho_{l}$ and $\mathbf{S}=-p_{l} \mathbf{I}$, where $p_{l}=\mathcal{P}\left(\rho_{l}\right)$, of mass density and stress tensor in liquid phase are attained asymptotically. As DPE implies $\lambda \rho_{v} \Delta \rho_{\rho=\rho_{v}}=\rho_{v}\left[\mu\left(\rho_{v}\right)-\mu\left(\rho_{l}\right)\right]$ we have: $p_{v}-p_{l}=W\left(\rho_{l}\right)-W\left(\rho_{v}\right)+\mu\left(\rho_{l}\right)\left(\rho_{v}-\rho_{l}\right)$ this difference is not equal to the difference of thermodynamical pressures as, for microscopic bubbles, $\mu\left(\rho_{l}\right)$ differs from $\mu\left(\rho_{v}\right)$. As the experimental results (see for instance [1]) deal with measures of stresses, then we have to use $p_{v}-p_{l}$ instead of $\mathcal{P}\left(\rho_{v}\right)-\mathcal{P}\left(\rho_{l}\right)$ in the comparing them with theoretical predictions. We can now define the surface tension and the radius of a bubble by identifying the nucleation energies and the pressure differences computed in Gibbs and second gradient:

$$
\begin{gathered}
p_{v}-p_{l}=\frac{2 \sigma}{R} \quad \text { and } \quad \frac{4}{3} \pi R^{2} \sigma=\frac{4}{3} \pi \int_{0}^{\infty} \lambda \rho_{r}^{2} r^{2} d r \quad \Rightarrow \\
R=\left[2 \lambda \int_{0}^{\infty} \rho_{r}^{2} r^{2} d r\right]^{\frac{1}{3}}\left[W\left(\rho_{l}\right)-W\left(\rho_{v}\right)-\mu\left(\rho_{l}\right)\left(\rho_{l}-\rho_{v}\right)\right]^{-\frac{1}{3}} \\
\sigma=\left[\frac{\lambda}{4} \int_{0}^{\infty} \rho_{r}^{2} r^{2} d r\right]^{\frac{1}{3}}\left[W\left(\rho_{l}\right)-W\left(\rho_{v}\right)-\mu\left(\rho_{l}\right)\left(\rho_{l}-\rho_{v}\right)\right]^{\frac{2}{3}}
\end{gathered}
$$

\section{Numerical solution of DPE.}

The DPE for the normalized density $\rho(r)$ reads (cf. $[6,11,12])$ :

$$
\rho_{r r}+\frac{2}{r} \rho_{r}=\mu(\rho)-\mu_{\infty}
$$

where subscript means that the derivatives are taken with respect to the normalized length variable $r$. Lengths and densities are normalized with respect: $\mathcal{L}=\sqrt{\frac{\mathcal{P}_{c}}{\lambda \rho_{c} Z_{c}}}$ and critical density. $\mu$ is the normalized chemical potential and $\mu_{\infty}=\mu\left(\rho_{l}\right) . Z_{c}$ and $\mathcal{P}_{c}$ are the critical compressibility ratio and pressure. We find a numerical solution of DPE, verifying the boundary conditions that imply the physically meaningful density profile describing the equilibrium of a bubble with its liquid: $\rho_{r}(0)=\rho_{r}(\infty)=0$. The numerical analysis is based on a 'mechanical' interpretation of Eq.(10) (cf. [13]). In fact DPE can be regarded as the equation of a 'particle' of mass one moving in the potential:

$$
U\left(\rho, \mu_{\infty}\right)=-\left(W(\rho)-\mu_{\infty} \rho\right)
$$

with the 'viscous' time-depending force $\lambda(2 / r) \rho_{r}$. We will call 'motion' of this particle as the solution of DPE starting from $r=0$, i.e., at the time zero, with zero velocity and with a given initial density, i.e., a given initial position, $\rho(0)$. From this consideration it is evident that a fundamental role in our problem is played by potential $U\left(\rho, \mu_{\infty}\right)$. Therefore before describing our numerical results we state some general considerations valid for any potential verifying the following hypotheses of 'biconvexity': in an interval $I=\left(\mu_{\infty}^{s p i, b u b}, \mu_{\infty}^{s p i, d r o p}\right)$ Eq.(11) have 
two maxima separated by a minimum. It exists a value $\mu_{\infty}^{s a t} \in I$ that separates bubble case from droplet case: for this value the interface is planar and the two maxima have the same height (Maxwell rule). For $\mu_{\infty}^{s p i, b u b}<\mu_{\infty}<\mu_{\infty}^{s a t}$ the maximum values of the potential in the high density (liquid) range for $\rho$ is always lower than that in the low density (vapor) range.

From such assumptions is clear that a 'motion' starting with initial position $\rho(0)$ in the region just at the right of the first maximum of the potential (bubble case) rolls down until the minimum and then goes up towards the second maximum. If the velocity is exactly that one sufficient to reach the second maximum (separatrix solution), then the 'particle' reaches the second maximum for $r$ tending to $\infty$; as at the maximum the velocity is zero, boundary conditions are verified. We will call $\rho_{2}\left(\mu_{\infty}\right)$ the value for $\rho$ in which the potential attains the second maximum. The force over the 'particle' is zero when $r$ tends to $\infty$ : from DPE we have $\mu\left(\rho_{2}\right)=\mu_{\infty}=\mu\left(\rho_{l}\right)$. This implies that (as we assume that locally for large values of density the function $\mu(\rho)$ is invertible) $\rho_{2}=\rho_{l}$.

\section{FIG.1}

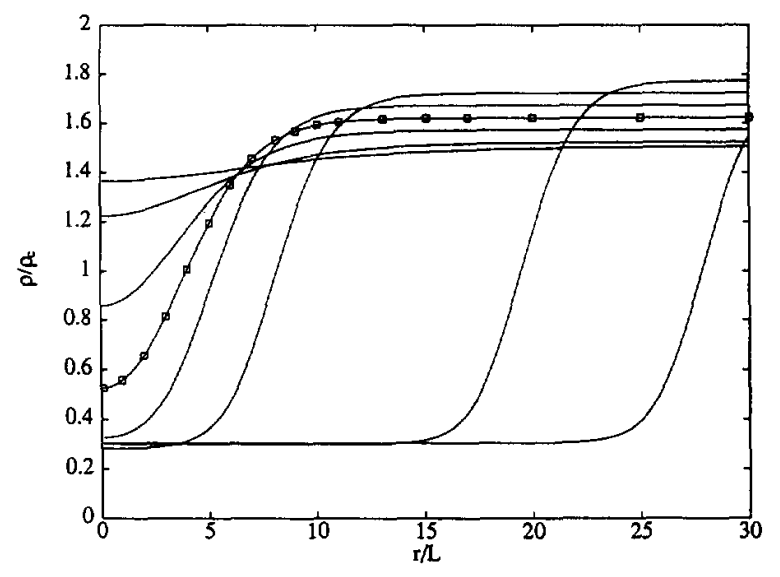

Numerically evaluated solution of DPE Eq.(14) for van der Waals equation of state for different values of $\mu_{\infty}$ at $T / T_{c}=0.85$. We note that near the minimal nucleation radius (the curve labelled by $\square$ ) the bulk phase vapor practically disappear.

So the solution of DPE corresponding to a phase transition is found if the 'motion' starting with an initial data $\rho(0)$ is exactly the separatrix one. So for a fixed $\mu_{\infty}$ the only unknown quantity is the 'initial data' $\rho(0)$ that we determine numerically solving DPE. We integrate this system by a Bulirsh-Stoer integrator [14] starting from an arbitrary guess value of $\rho(0)$ with $\rho_{r}(0)=0$. The values of $\rho(0)$ are then adapted with a sequence of integrations until a good approximation to the separatrix is reached. At the saturation the accuracy of the method is limited by the machine precision because the starting density differs from first maximum density by progressively smaller quantities. We were limited by the usual double precision $\left(10^{-16}\right)$ of our computing device. This precision is sufficiently high to catch the main features of the approach to the saturation. Typical density profiles are shown in Fig.1 for the van der Waals equation of state. The homogeneous nucleation and the growth of the 
bubble approaching the saturation, are clearly seen in this figure. The density profile tends to be diffuse towards the spinoidal limit and the density jump $\rho_{\infty}-\rho(0)$ goes to zero.

FIG.2

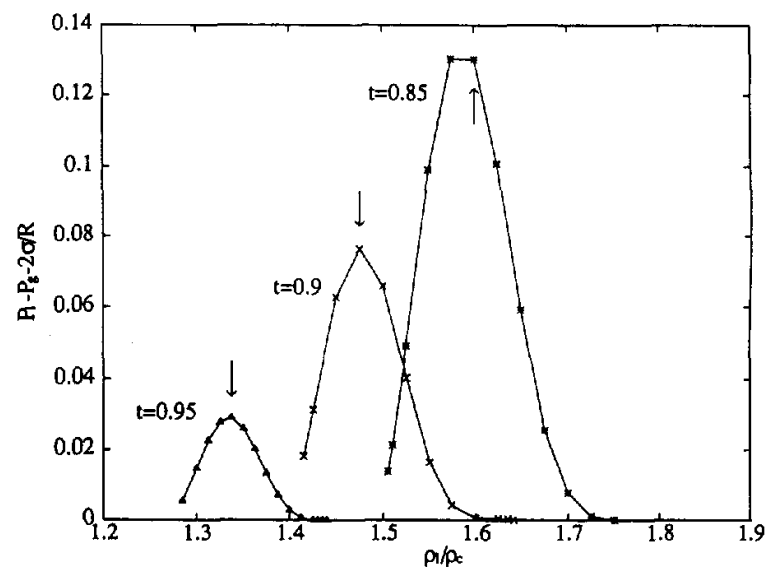

Departure from classical Laplace formula in van der Waals small bubbles at $T / T_{c}=0.85$, 0.9 and 0.95 . Pressure difference on $y$-axis is normalized to critical pressure $\mathcal{P}_{c}$; the arrows marks the points corresponding to the minimal nucleation radius.

Approaching the saturation a region of bulk vapor phase appears before the phase transition region and the density jump is practically constant. In Fig. 2 we plot the departure from the classical Laplace formula for three reduced temperatures as function of $\rho_{\infty}$. The departure have a maximum very close to the minimal nucleation radius (cf. Fig.3 below) and can reach significative fractions $(10 \%)$ of $\mathcal{P}_{c}$ just for $T / T_{c}=0.85$.

FIG.3

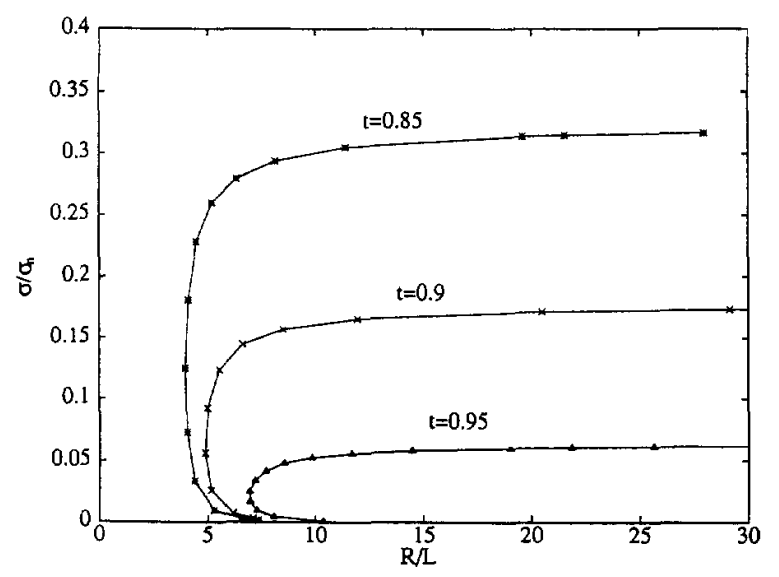

Normalized surface tension $\sigma / \sigma_{n}$ with $\sigma_{n}=\rho_{c} R_{\mathcal{M}} T_{c} \mathcal{L}$ vs normalized equilibrium radius $R / \mathcal{L}$ of van der Waals bubbles at $T / T_{c}=0.85,0.9$ and 0.95 . 
In Fig.3 we show the dependence of $\sigma$ on $R$ numerically evaluated by Eq.s(9) at the same reduced temperatures of Fig.2. The results are qualitatively similar: for small values of $\mu_{\infty}$ (i.e. the lower part of the curve) $\sigma$, initially vanishing, grows slowly whereas the radius decreases towards a minimal value that we identify as the minimal nucleation radius $R_{m}$. At $R_{m}$ the surface tension suddenly increases and the density at the center of the bubble reaches the range of the vapor phase (cf. also Fig.1). Above the minimal nucleation radius $\sigma$ reaches within few minimal radii the planar interface value and as shown by experiments [1] remains substantially constant.

\section{Acknowledgments}

We gratefully thank prof. H.Gouin for having discussed with us the content of this paper and for his wise advice. We thank prof. A.Di Carlo and MCD P.Seppecher for their criticism.

† Permanent address: Dipartimento di Energetica, Facoltà di Ingegneria,

Università dell'Aquila, Roio Poggio 67040, L'Aquila, ITALY.

1. L.R.Fisher and J.N.Israelachvili, Chem.Phys.Lett.76, 325 (1980);

2. P.Germain, J.de Mecanique, 12, 235 (1972);

3. Dell'Isola et al., C.R.Acad.Sci.Paris in print (199?);

4. J.W.Gibbs, Collected works, vol.1, Yale Univ. Press (1948);

5. R.C.Tolman, J.Chem.Phys.17, 333 (1949);

6. P.Casal, Cahier du groupe Francais de rhéologie, CNRS VI, 3, 31 (1961)

7. P.Casal, C.R.Acad.Sci.Paris 274, Série A, 1571 (1972);

8. P.Casal and H.Gouin, C.R.Acad.Sci.Paris 300, Série II, 231 (1985);

9. P.Seppecher, These, Université Paris VI and E.N.S.T.A.;

10. Y.Rocard, Thermodynamique, Masson, Paris (1967);

11. A.Blinowski, Arch.Mech.(Warsaw), 26, 953 (1974)

12. J.W.Cahn and J.E.Hilliard, J.Chem.Phys.28, 258 (1958); ibid.31, 688 (1959);

13. N.G.Van Kampen, Phys.Rev.135, A362 (1964);

14. W.H.Press et al., Numerical Recipes, Cambridge Univ. Press, Cambridge (1986); 